where $a$ is an arbitrary constant and $\chi(\rho)$ an arbitrary differentiable function of $\rho$. It is found to be a transform of Schwarzschild's external solution :

$$
\begin{gathered}
d s^{2}=\left(1-\frac{2 m}{r}\right) d t^{2}-\left(1-\frac{2 m}{r}\right)^{-1} d r^{2}- \\
r^{2}\left(d \theta^{2}+\sin ^{2} \theta d \varphi^{2}\right) .
\end{gathered}
$$

If we put $\alpha=2 m$ and $\chi(\rho)=1$, (6) reduces to

$$
\begin{aligned}
d s^{2}= & d \tau^{2}-\left[1 \pm \frac{3}{2} \frac{\sqrt{2 m} \tau}{\rho^{3 / 2}}\right]^{-2 / 3} d \rho^{2}- \\
& \rho^{2}\left[1 \pm \frac{3}{2} \frac{\sqrt{2 m \tau}}{\rho^{3 / 2}}\right]^{4 / 3}\left(d \theta^{2}+\sin ^{2} \theta d \varphi^{2}\right), .
\end{aligned}
$$

which is connected with (7) through the transormation,

$$
\begin{gathered}
r=\rho\left[1 \pm \frac{3}{2} \frac{\sqrt{2 m} \tau}{\rho^{3 / 2}}\right]^{2 / 3}, \\
t=\tau-\tau_{0} \pm 2 \sqrt{2 m}\left(\rho^{3 / 2} \pm \frac{3}{2} \sqrt{2 m \tau}\right)^{1 / 3} \pm \\
2 m \log \frac{\left(\rho^{3 / 2} \pm \frac{3}{2} \sqrt{2 m \tau}\right)^{1 / 3}-\sqrt{2 m}}{\left(\rho^{3 / 2} \pm \frac{3}{2} \sqrt{2 m} \tau\right)^{1 / 3}+\sqrt{2 m}},
\end{gathered}
$$

$\tau_{0}$ being an arbitrary constant. The Jacobian of the transformation is

$$
\frac{\delta(r, t)}{\delta(p, \tau)}=\left(\frac{p}{r}\right)^{1 / 2}
$$

Comparing (7) with (8), we find that the former gives flat space when $m=0$ or as $r \rightarrow \infty$, while the latter does so when $m=0$ or $\tau=0$ or as $\rho \rightarrow \infty$. The form (8) requires $m$ to be positive. assume that $n$ is positive. The condition $(3)$

$r \rightarrow \infty$, while it is true everywhere for $(8)^{2}$. elsewhere.

Department of Mathematics,
Benares Hindu University.

V. V. NARIIKAR.

K. R. KARMARKAR. Dec. 29.

1 Eisenhart, L. P., "Riemannian Geometry, 57 (1926),

"Weyl H, "Space, Time and Matter", 275 (1922).

' Narlikar,'v. V., Mon. Not. Roy. Ast. Soc., 93, 263 (1936)

\section{A Simple Proof of the Lorentz Transformation}

IT appears that the following proof of the Lorentz transformation, which occurred to me when preparing a lecture on relativity, is not generally known among students of relativity, though $I$ would be surprised if it has not been discovered before.

Let $\Sigma(x, y, z, t)$ and $\Sigma^{\prime}\left(x^{\prime}, y^{\prime}, z^{\prime}, t^{\prime}\right)$ be two systems of reference in uniform motion with respect to one another. Let the $x$ - and $x^{\prime}$-axis be in the direction of motion. Let $v$ he the velocity of $\Sigma^{\prime}$ measured in $\Sigma$ Its value depends on the scales adopted in $\Sigma$ for the measurement of length and time and also on the deflnition of synchronism used in $\Sigma$ for events in $\Sigma$. We on the that the scales and the definition of synchronism used in $\Sigma^{\prime}$ are such that the velocity of $\Sigma$ measured in $\Sigma^{\prime}$ has the value $-v$.

It then follows that the transformation formulæ are

$$
\begin{aligned}
& y^{\prime}=y, z^{\prime}=z \\
& x^{\prime}=f_{11}(v) x+f_{12}(v) t
\end{aligned}
$$

and that the transformation matrix

fulfils the postulate

$$
F(v)=\left(\begin{array}{ll}
f_{11}(v) & f_{12}(v) \\
f_{21}(v) & f_{22}(v)
\end{array}\right)
$$

$$
F^{\prime}(-v)=F(v)^{-1} \text {. }
$$

Let now $\Sigma^{*}\left(x^{*}, y^{\prime \prime}, z^{\prime \prime}, t^{\prime \prime}\right)$ be a third system of reference with its $x^{\prime \prime}$-axis parallel to the $x^{\prime}$-axis and moving along the latter with the
uniform velocity $v^{\prime}$ as measured in $\Sigma^{\prime}$. The matrix of the transformauniform velocity $v^{\prime}$ as measured in $\Sigma^{\prime}$. The matrix of the transformation $\Sigma \rightarrow \Sigma^{\prime \prime}$ is then the product matrix $F\left(v^{\prime}\right) F^{\prime}(v)$. If, on the other hand, we introduce the intermediate System $\Sigma^{\prime}$ so that its velocity tion is given by $F(v) F\left(v^{\prime}\right)$. Hence

$$
F(v) F^{\prime}\left(v^{\prime}\right)=F\left(v^{\prime}\right) F^{\prime}(v) \text {. }
$$

The two postulates $(P 1)$ and $(P 2)$ together with the definition of the group parameter $v$ suffice to evaluate the four functions $f_{i k}(v)$.

By writing down the four equations $(P 1)$ and hence forming the determinant $D(-v)=\operatorname{det}[F(-v)]$ it is seen that $D(-v)=D(v) / D(v)=$ 1. Hence

$$
D(v)=\operatorname{det}[F(v)]=1
$$

The four equations $(P 1)$ then reduce to $\begin{array}{lll}f_{11}(-v)=f_{22}(v)(2 \cdot 11) & f_{12}(-v)=-f_{12}(v)(2 \cdot 12) \\ f_{21}(-v)=-f_{21}(v)(2 \cdot 21) & f_{22}(-v)=f_{11}(v)(2 \cdot 22)\end{array}$ of which the first and the last are identical. To these equations $(P 2)$ adds one, and only one, new functional equation :

$$
f_{12}(v) / f_{21}(v)=f_{12}\left(v^{\prime}\right) / f_{21}\left(v^{\prime}\right)=\text { invariant }=C^{2} .
$$

The results so far obtained may be summarized as follows:

$$
F(v)=\left(\begin{array}{ll}
f_{11}(v) & f_{12}(v) \\
f_{12}(v) / C^{2} & f_{11}(-v)
\end{array}\right), f_{12}(-v)=-f_{12}(v) \text {. }
$$

We now make use of the deflnition of $v$ as the velocity of $\Sigma^{\prime}$ in $\Sigma$, that is, $v=x / t$, where $x$ and $t$ are corresponding co-ordinates in $\Sigma$
of the point $x^{\prime}=0$. Hence from $(1 a)$ :

$$
v=-f_{12}(v) / f_{11}(v)
$$

Since $f_{12}(v)$ is an uneven function of $v$, it follows that $f_{11}(v)$ is an even function :

$$
f_{11}(-v)=f_{11}(v) \text {. }
$$

Hence the matrix $F(v)$ reduces to

From (1) we obtain

$$
F(v)=f_{11}(v) \cdot\left(\begin{array}{cr}
1 & -v \\
-v / C^{2} & 1
\end{array}\right) .
$$

$$
f_{11}(v)=\left[1-v^{2} / C^{2}\right]^{-1 / 2} \text {. }
$$

From the last formula, or more convincingly from the theorem of composition of velocities, it is seen that the invariant $C$ is the limiting velocity.

Since the postulates $(P 1)$ and $(P 2)$ can always be satisfied by a suitable choice of the definitions involved, it appears that the Lorentz transformation has a more general significance than seems to be widely believed. Indeed, the only question left for the experimenter to decide is what signals have the limiting velocity $C$, and what is its value in a given system of units. In particular, the Galilean transformation does not logically contradict the Jorentz transformation but is its degenerated form for $C=\infty$. In order to establish the Special Theory of Relativity, no other empirical fact need be used than that for any given system of reference the velocity of light is the limiting velocity.

Woolwich Polytechnic,
Iondon, S.E.18. Jan. 3.

M. D. H. Strauss.

\section{A Generalization of Special Relativity Theory}

IN an attempt to treat gravitational and electromagnetic phenomena simultaneously within the framework of the special theory of relabe generalized to five dimensions and derivatives with respect to the fifth co-ordinate equated to zero. One may similarly generalize to five dimensions with special relativistic dynamics of a continuous medium, identifying the extra components of the mechanical stress medium, identifyi

$$
T^{\mu s}=c j, c^{2} \rho, c^{8} \sigma_{0},
$$
where $j, \rho, \sigma_{0}$ denote the current, charge and rest-mass densities, Assuming that this macroscopic tensor may be added to the corre associated with a gravito-electromagnetic fleld there exist charge and current densities given by

$$
c j(f)=\mathbf{H} \times \mathbf{F}-\theta \mathbf{E} ; \quad c^{2} \rho(f)=-\mathbf{E} \cdot \mathbf{F} ;
$$

where $F=-\nabla \Omega, c \theta=\dot{\Omega}$, and $\Omega$ is the gravitational potential of the consequent scalar gravitational theory.

These equations imply that mass and momentum, but not charge, may be created from the fleld at points unoccupied by matter, and that, although charge may be carried by the fleld, the net charge radiated per unit time over a distant boundary by an accelerated charge vanishes identically. It also follows that the charge and current densities in Maxwell's equations should include $\rho(f)$ and $j(f)$ respectively, so that in general they are non-zero even in material-free space. to be functions of the gravitational potential :

$$
m_{0}=m_{0 \infty} \exp \left(\Omega / c^{2}\right) ; \quad e=e_{\infty} \exp \left(\Omega / c^{2}\right) .
$$

Hence the force between two particles tends to zero as they approach each other indefinitely.

The assumption of flat space-time with

$$
\frac{\partial}{\partial x^{5}}=0
$$

yields a scalar gravitational theory similar to the earlier theory of Nordström ${ }^{2}$ and leaves out of account the remarkable successes of the general theory of relativity. The results to which the above theory leads should therefore be treated with caution. Nevertheless, one is thus able to formulate a relativistically covariant special theory from which the infinite forces of classical electromagnetic theory are absent.

Department of Physics,

$$
\begin{aligned}
& \text { University of Melbourne. } \\
& \text { Jan. } 8 \text {. }
\end{aligned}
$$

H. C. CORBEN. Jan. 8.

1 Nordström, G., Phys. Z., 15, 504 (1914). Corben, H. C., Nature,

156,388 (1945).
2 Nordström, G., Phys. Z., 13, 1126 (1912). 